\title{
A microprocessor-aided platform enabling surface differential reflectivity and reflectance anisotropy spectroscopy
}

\author{
Gianlorenzo Bussetti $^{1, \mathrm{a}} \mathbb{1}$, Lorenzo Ferraro ${ }^{1}$, Alberto Bossi ${ }^{2,3}$, Marcello Campione ${ }^{4}$, \\ Lamberto Duò $^{1}$, Franco Ciccacci ${ }^{1}$ \\ ${ }^{1}$ Dipartimento di Fisica, Politecnico di Milano, p.za Leonardo da Vinci 32, 20133 Milan, Italy \\ 2 Istituto di Scienze e Tecnologie Chimiche "G. Natta" of the CNR (CNR-SCITEC), via Fantoli \\ 16/15, 20138 Milan, Italy \\ ${ }^{3}$ SmartMatLab Center, via Golgi 19, 20133 Milan, Italy \\ ${ }^{4}$ Department of Earth and Environmental Sciences, Università degli Studi di Milano - Bicocca, Piazza della \\ Scienza 4, 20126 Milan, Italy
}

Received: 10 November 2020 / Accepted: 23 March 2021

(C) The Author(s) 2021

\begin{abstract}
Surface differential reflectivity (SDR) and reflectance anisotropy spectroscopy (RAS) [sometimes known as reflectance difference spectroscopy] are two well-known optical spectroscopies used in the investigation of surfaces and interfaces. Their adaptability on different experimental conditions (vacuum, controlled atmosphere and liquid environment) allows for the investigation not only of surface states and/or ultra-thin films but also of more complex interfaces. In these circumstances, the analysis of the sample with both techniques is decisive in view of obtaining a correct picture of the sample optical properties. In this work, we show a microelectronic hardware solution useful to control both a SDR and a RAS apparatus. We describe an electronic architecture that can be easily replicated, and we applied it to a representative sample where the interpretation of the optical properties requires an analysis by both SDR and RAS.
\end{abstract}

\section{Introduction}

Nowadays, light probes are successfully applied in semiconductor technology [1-3], surface physics [4-6], chemical detection [7-9], biological analysis [10-12], etc. More specifically, the surface and interface physics raised great interest in the optical characterization of layered systems [13-15] and molecular films [16-18]. This is due to the adaptability of optical spectroscopy set-ups to work in different conditions [19] and to the possibility to investigate also buried interfaces [20] when chemical processes are in progress [21]. The application of an optical spectroscopy, namely surface differential reflectivity (SDR or DRS), to the detection of surface states must be dated back to 1976, when P. Chiaradia and co-workers reported the first direct evidence of optical transition inside the band gap of a semiconductor [22]. Generally speaking, SDR compares the sample reflectivity under two different physical situations, e.g., the freshly prepared sample and after the surface passivation or when an

\footnotetext{
a e-mail: gianlorenzo.bussetti@polimi.it (corresponding author)
} 
electric field is applied or not to the specimen, etc. Another strategy foresees the acquisition in parallel of reflected light from the sample under investigation, and from a so-called dummy sample, the latter only used for the signal normalization. Since 1976, SDR was applied to study the optical properties of different semiconductor surfaces [23-25], the role of molecular adsorption in quenching surface states [26], the optical properties of organic films [27, 28], etc. SDR can also exploit polarized light. In this configuration, it was used to confirm the Pandey's chain model for the Si(111) $(2 \times 1)$ reconstruction [29]. In fact, Pandey's chains break the surface isotropy and show a different reflectivity if light is polarized along the chains or in the orthogonal direction. The $\operatorname{Si}(111)(2 \times 1)$ IR optical transition can be measured also by using the reflectance anisotropy spectroscopy (RAS or RDS) set-up [30]. In RAS, the sample is shined by a linearly polarized light modulated $10^{5}$ times/s between two orthogonal directions. As a consequence, RAS does not foresee the comparison between two sample physical states or between the specimen under investigation and a dummy sample. By exploiting the polarized light modulation, this spectroscopic technique can directly detect a signal if sample shows anisotropy. With respect to SDR, the RAS high working frequency, the use of an optical modulator and a low-noise photomultiplier also ensure a better experimental stability, a better signal-to-noise ratio and the possibility to detect very small signal to few parts of $10^{-6}$ of the total reflected beam [31]. By using an optical modulator (namely photoelastic modulator or PEM), RAS directly detects the relative sample anisotropy between two orthogonal directions. SDR, when instead used with polarized light, can measure each contribution in polarization: the sample anisotropy thus arises only after a subtraction between spectra. RAS was originally applied on $\mathrm{Si}(110)$ by Aspnes and co-workers in 1985 [32]. Since then, RAS was successfully applied on similar systems explored by SDR but, with respect to the latter, also on more complex organic films [33, 34], solid-liquid interfaces [35, 36] or for volatile compounds detection [37]. Both SDR and RAS spectra can be interpreted within the three-layer model, which considers the surface, or a film grown onto a substrate, as an absorbing layer with its own dielectric constant [38]. The model is predictive if the film thickness is well below the light wavelength used in the experiment. Generally, both SDR and RAS work in the $250 \mathrm{~nm} \div 4150 \mathrm{~nm}$ spectral range. Consequently, ultra-thin film (within few nanometers) well satisfy the three-layer model hypothesis.

A notable occurrence in RAS is the collection of spectra showing sometimes particular features, whose behavior is referred to as "derivative-like" line shape, where a direct correlation between the RAS signal and the main absorbance peak is not straightforward. An emblematic case is offered by RAS spectra collected on porphyrin (an organic molecule) Langmuir-Blodgett or Langmuir-Schaefer films [39]. The complex stratified porphyrin film structure, where molecule optical transitions can undergo small changes due to differences between the layers, can produce a spectrum that is not easily related to the porphyrin absorbance spectrum and the film anisotropy [40]. We posit that, in these cases, a comparison between RAS spectra and SDR measurements (with and without polarized light) is crucial for a correct interpretation of data.

With the aim of offering the possibility to build up versatile SDR and RAS systems to researchers involved in the investigation of the optical properties of stratified films, buried and solid-liquid interfaces (i.e., cases where the light beam must pass different media), we show and discuss an electronic architecture that can be easily replicated and used in laboratory. To prove the opportunity of comparing SDR and RAS spectra, we present an analysis of the optical properties of an emblematic system: meso-tetraphenyl porphyrin $\left(\mathrm{H}_{2} \mathrm{TPP}\right)$ thin film grown on highly oriented pyrolytic graphite (HOPG). In this system, RAS reveals an apparently anomalous redshift of the main porphyrin optical transition. We show that this can 
be explained and rationalized in terms of a proper combination of unpolarized and polarized SDR measurements.

\section{SDR and RAS set-ups}

\subsection{SDR}

We propose a double-channel SDR set-up where, instead of the traditional Michelson cross [41], we exploit branched optical fibers and an optical switch. In Fig. 1, we report the used experimental configuration.

The light source (DH-2000-Bal, Ocean Optics) is a combined deuterium-halogen lamp suitable to cover the entire visible and near-UV spectral range. A technical solution adopted by Ocean Optics ensures a good light intensity stability as a function of time. A branched optical fiber (Avantes FCB-UVIR400-2-ME) allows to shine light on two targets in parallel. The first one is the real sample (in our experiment a porphyrin film deposited on HOPG), while the second one is a fictitious sample (dummy) used for the signal normalization. Here, the dummy sample is a clean HOPG substrate. The reflected light (under a small angle of few degrees) from both the sample and the dummy one is collected in two fibers linked with an optical switch. The latter is connected with an optical multichannel spectrometer. The optical switch (Avantes, FOS-2-INL) allows to detect alternately signals coming from the sample

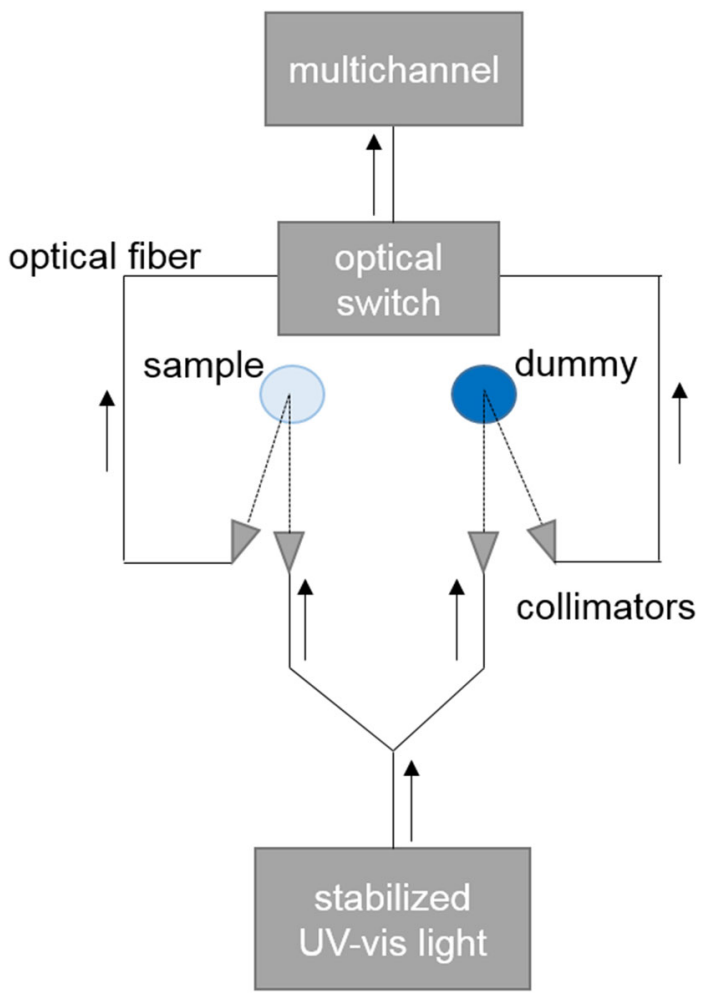

Fig. 1 a Schematic of the SDR set-up 
( $\left.R_{\text {sample }}\right)$ and the dummy $\left(R_{\text {dummy }}\right)$ at a frequency of about $5 \mathrm{~Hz}$. The percentage SDR signal is defined as:

$$
10^{2}\left[\left(R_{\text {sample }}\right)-\left(R_{\text {dummy }}\right)\right] /\left(R_{\text {dummy }}\right)
$$

The acquired signal is further averaged by the software. The SDR calibration was obtained by measuring clean HOPG (sample equivalent to dummy), and the results are reported below.

A particular care must be devoted to the light input from the source to the fiber and from the fiber to the detector (in our case we employed an OCEAN-HDX-XR): small misalignments can produce a significant background. We experimentally observed that when the two optical paths are compared on the same system (dummy = sample) the background can show wide bumps having an intensity of some units in $10^{-2}$. This situation is even more critical when twin fibers, closely embedded together, are employed in the SDR apparatus. In this case, the original factory coupling can reach its limit: a small change in how light impinges on the optical fiber core introduces inequalities between the two optical paths. In fact, in this case, the source spectrum is clearly different. To overcome this problem some strategies can be exploited, according to the required detection limit. For example, one or two optical condensers (i.e., a system of lenses that acts to properly focus the light beam) can be placed within the light source and the optical fiber and/or the latter and the spectrometer. For our measurements on organic films, we followed a different approach: working with twin fibers, the main problem was their alignment that must be parallel to the spectrometer multichannel array. For this reason, we spent time to precisely find the correct position of the fiber inside the SMA connector by turning the fiber cable. During this operation, the SDR signal was monitored in real time and the fiber was tightened only when the SDR background was reasonably minimized.

\subsection{RAS}

In Fig. 2, we report a sketch of the RAS set-up. We adopt the same DH-2000-Bal light source, but it is not strictly necessary. Xe arc lamps with high brilliance are also often employed in many RAS apparatuses.

The RAS apparatus has two polarizers (Glan-Taylor or Glan-Thomson). The second one along the light path is called analyzer. Differences in working with one or two polarizers are discussed in Ref. [42]. With small changes in the RAS optical configuration, a system of lenses of different focal lengths and provided by ThorLabs can be added to properly focus the light beam on the sample and/or on other optical devices, or even better mirrors (not suffering from wavelength depending focusing) can be efficiently employed. The light polarization is rotated along two orthogonal directions $(\alpha$ and $\beta$ ) by the photo-elastic modulator (PEM-100, provided by Hinds). The angle of reflection $(\Theta)$ is generally of few degrees. The reflected light reaches a monochromator (Spectral Products CM 110) and is finally detected by a multialkali photomultiplier (Hamamatsu R 1463). Being the light polarization related to the PEM retardation, the overall reflectivity difference is a complex value as reported in Ref. [43]. However, if only the real part is measured, a noticeable relation occurs between the complex reflectance term and the reflectivity coefficient $\left(2 \operatorname{Re} \Delta r / r=2\left(R_{\alpha}-R_{\beta}\right) /\left(R_{\alpha}+R_{\beta}\right)\right]$. The RAS signal can be thus directly defined as:

$$
2\left[\left(R_{\alpha}\right)-\left(R_{\beta}\right)\right] /\left[\left(R_{\alpha}\right)+\left(R_{\beta}\right)\right]
$$

The reflectivity difference is directly measured by a lock-in amplifier with the reference provided by the PEM. Details in the RAS signal interpretation can be found in Ref. [43]. 


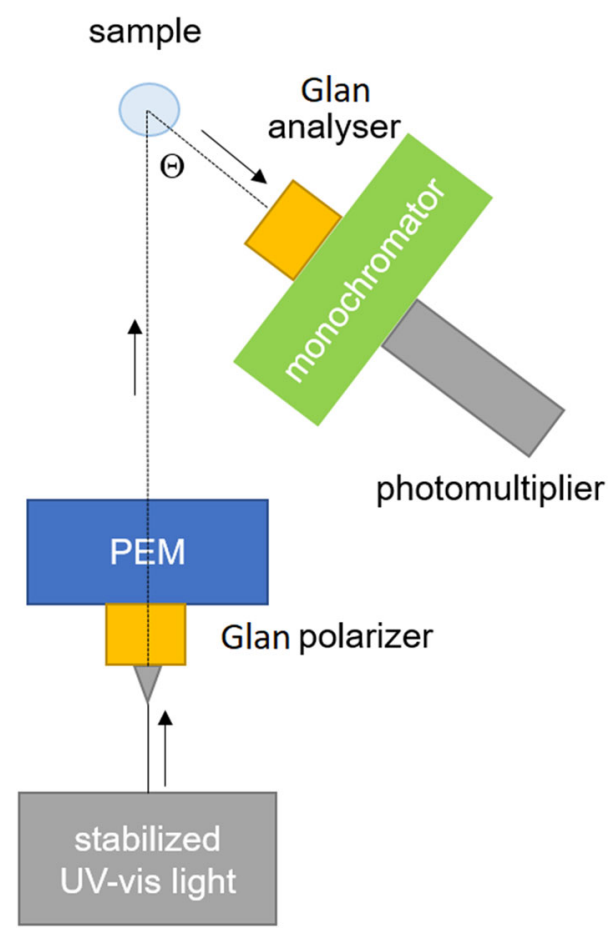

Fig. 2 a Schematic of the RAS set-up

Here, we observe that, when organic films are analyzed, the alignment of the sample with respect to the PEM $\alpha$ and $\beta$ directions is a priori unknown. An azimuthal analysis is thus required [44].

The full system has been calibrated by measuring spectra from two different silicon surfaces, namely $\mathrm{Si}(110)$ and $\mathrm{Si}(001)$, the first yielding a well-known RAS signal [32] and the second being representative of an almost isotropic sample [45].

Finally, we note that the possibility of combining both SDR (which measures all the sample optical transitions) and RAS (which is sensible to the anisotropic optical transitions) for the analysis of the optical properties of a surface recalls, in a certain way, what can be measured by a Mueller matrix ellipsometer. The latter allows for the dielectric (anisotropy) function determination and also depolarization effects.

\subsection{The electronic architecture}

In Fig. 3, we report a block diagram for the instrumentation control.

The spectroscopic signal, acquired from the optical set-up, comes out from the multichannel spectrometer, in the SDR system, or from the photomultiplier in the RAS apparatus. The main control system consists of a microprocessor (ESP32 espressif) that can directly read (i) the multichannel output or (ii) the photocurrent generated from the photomultiplier. Regarding (i), the main control uses a clock to drive the optical switch and to alternatively acquire the spectrum coming from the sample and the dummy one, respectively. According to the final integration time, the signal of the two channels is read many times to quench both fast and slow signal instabilities. A good compromise is an integration time of $\tau=90 \mathrm{~s}$. If the RAS is employed (ii), the ESP32 espressif microprocessor must drive the photomul- 

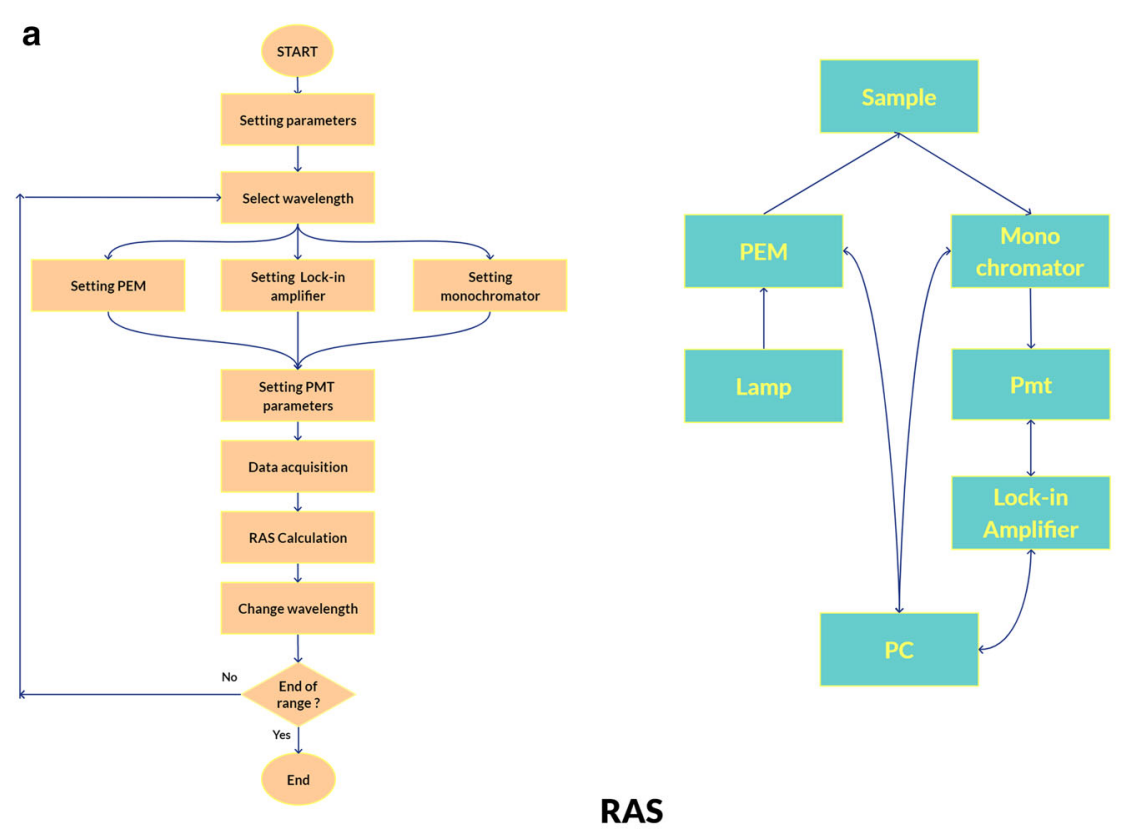

\section{RAS}

b

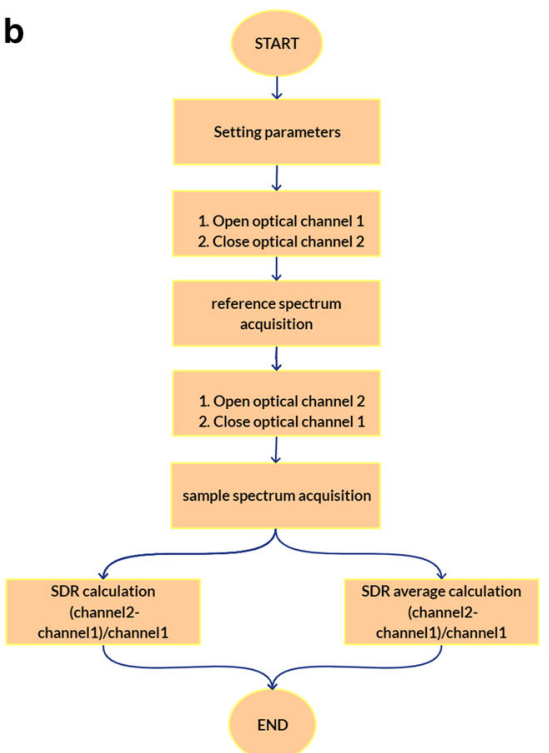

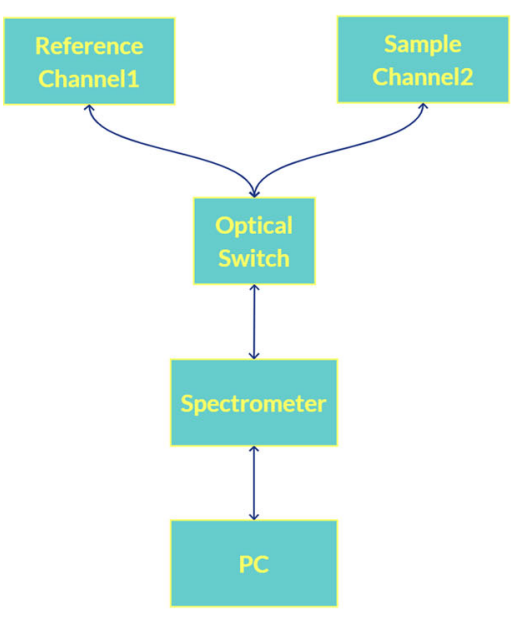

\section{SDR}

Fig. 3 RAS (a) and SDR (b) block diagram for instrumentation control

tiplier. In this case, the signal coming from the detector first passes through a $2 \times 10^{4} \mathrm{~V} / \mathrm{A}$ transimpedance amplifier and then is filtered and converted into a digital signal by a 16-bit ADC converter (MAX1169 MAXIM). After digital conversion, the signal is acquired by the main control system software, which uses it for the photomultiplier power supply voltage feedback control. The latter is mandatory to bring the photomultiplier to the voltage values 
a

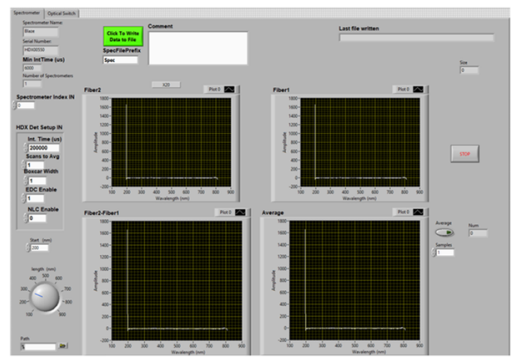

b

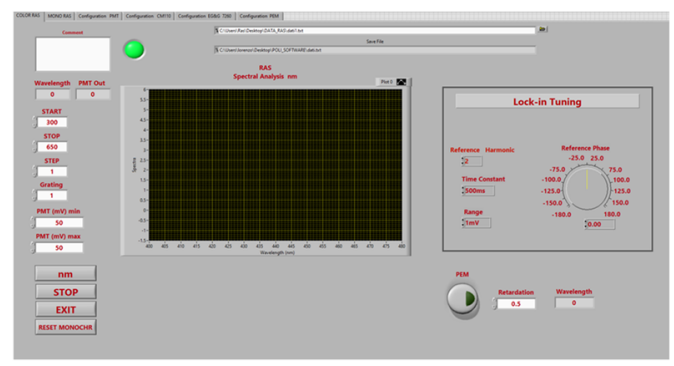

Fig. 4 Screenshots of the SDR (a) and RAS (b) control panel

interval where its performances are optimized, and the electronic noise is minimized. The feedback is simply managed by a software PID (proportional integrative derivative) applied to the power supply of the photomultiplier. The main control system also drives PEM and the lock-in amplifier. Generally, it seems that a lower signal noise is observed if the used reference signal, generated by the PEM control unit, is the natural piezo-resonance $(50 \mathrm{kHz})$ and the lock-in works in second harmonic $(100 \mathrm{kHz})[42]$.

\subsection{The acquisition program}

LabView is used to drive both SDR and RAS apparatus and to collect spectra. In Fig. 4, we show a screenshot of the SDR (panel $a$ ) and RAS (panel $b$ ) control panel.

SDR requires four acquisition screens for a complete evaluation of the signal. The two ones on the top refer to the sample and dummy channels, respectively. At the bottom, a first screen displays the SDR signal, as defined in Eq. (1), while the second one plots an averaged SDR spectrum after $N$ cycles. We define cycle a comparison between the spectrum arising from the sample and that one collected from the dummy. The sample (dummy) signal is integrated according to what the operator selects on the virtual trimmer at the bottom left of panel $a$. The number $N$ can be instead inserted in the blank space placed at the right. Other possible controls are useful for selecting the spectral range, parameters of the used detector or for typing some notes and the file name. A second LabView window (not shown) is only devoted to drive the optical switch. This operation is mandatory during the system calibration and the possibility of exploiting a special window really helps in the preliminary operations before the spectrum acquisition.

In panel $b$, we show the RAS control panel. In this case, different windows are present to drive the monochromator, the lock-in amplifier, the PEM device and, in particular, to acquire the RAS signal at fixed wavelength [mandatory during the growth of thin films in molecular beam epitaxy (MBE) or organic-MBE (OMBE) systems]. In the figure, we report the main acquisition window. As well as for the SDR program, the user can insert comments, the file name and select the preferred spectral range. In addition, the spectral step (minimum value $0.1 \mathrm{~nm}$ ) is optional in the program. The photomultiplier parameters (minimum and maximum photocurrent) can be put in the white boxes on the left. The grey square on the right instead gethers the lock-in settings (harmonic, time integration, sensitivity, dB gain, etc.).

Finally, both programs allow to superimpose different spectra on the same screen: in this way, the user can directly pinpoint differences during the spectrum acquisition. ${ }^{1}$

1 Researchers interested in building up their own SDR and RAS systems, are encouraged to directly contact the corresponding author by e-mail in view of having the detailed electric schemes as well as the LabView 


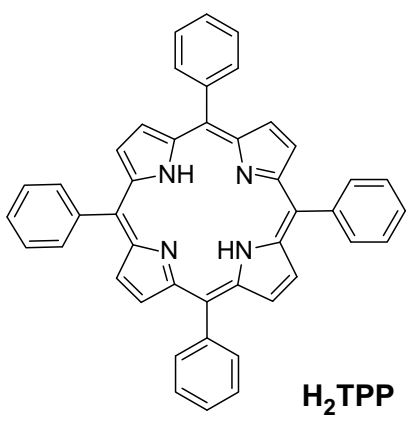

$12.5 \AA$

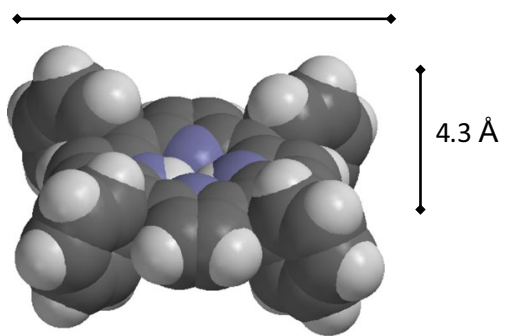

Fig. 5 (left) Planar structure of the 5, 10, 15, 20 tetraphenyl porphyrin $\mathrm{H}_{2} \mathrm{TPP}$ (one possible tautomer); (right) 3D-space-filling model displaying the molecular occupancy (aspect ratio of about $12.5 \AA$ wide by $4.3 \AA$ height ground state optimized geometry at the DFT B3LYP/6-31 g** level)

\subsection{Sample preparation and preliminary characterization}

HOPG is a synthetic type of graphite with an angular spread in the stacking of its graphene sheets and crystallites of less than $2^{\circ}$, and it is widely used in basic research because of its high purity [46-48]. HOPG is a highly stable and inert material (up to $3000{ }^{\circ} \mathrm{C}$ under vacuum), its atomically flat (0001) cleavage surface may serve as a calibration standard [49]. In these experiments, HOPG (Optigraph) has been exfoliated two times (by tape peel-off) prior to any analysis or its use as substrate for the epitaxial growth of $\mathrm{H}_{2} \mathrm{TPP}$. The latter was purchased by Sigma-Aldrich (99\%) and used without further purification.

$\mathrm{H}_{2}$ TPP (Fig. 5) is a planar hetero-macrocycle which consists of four pyrrole rings linked at their $\alpha$-positions by methin groups $\left(=\mathrm{CH}-\right.$ ). The conjugated $\pi$-system of $\mathrm{H}_{2} \mathrm{TPP}$, and of porphin in general, contains 18 electrons along the shortest cyclic path thus obeying the Hückel rule for aromatic systems. The two pyrrolic hydrogen atoms (-NH-) are subjected to tautomerism based on an intramolecular double proton transfer [50, 51].

The introduction of functional groups (FGs) on the methin position or on the free pyrrole ones lead to an almost infinite number of possible porphyrin derivatives that can be engineered for different scopes and applications. FGs on meso-positions, because of their sterical hindrance, are not allowed to be coplanar to the tetra-pyrrole core (Fig. 5); hence, porphyrins tend to undergo a saddle distortion of the macrocycle in order to favor planarization, thus extending the conjugation over these FGs.

$\mathrm{H}_{2}$ TPP, due to the conjugated and rigid core, displays optical absorptions in visible range between 390-430 $\mathrm{nm}$ (Soret band with molar absorptivity $\varepsilon>10^{6} \mathrm{M}^{-1} \mathrm{~cm}^{-1}$ ) followed by a set of smaller transition between 500-700 nm (Q-bands) in solution (Fig. 6, black and dashed gray lines).

$\mathrm{H}_{2}$ TPP films (5.0 nm thick) were grown by physical vapor deposition (PVD) at a base pressure of $5 \times 10^{-6}$ mbar. The growth chamber (Kenositec KE-500) includes four Knudsen cells (K-cell) and two thermal sources. The sample holder is positioned $30 \mathrm{~cm}$ away from the K-cell. The K-cells are thermally controlled via a thermocouple; growth was conducted between $320-330{ }^{\circ} \mathrm{C}$ at a flux of $0.11-0.17 \AA$ Às. Prior the deposition, $\mathrm{H}_{2}$ TPP K-cell was allowed to warm at $160^{\circ} \mathrm{C}$ for $30 \mathrm{~min}$ for outgassing.

programs for driving the apparatus. If our work would be useful for some researchers, we will appreciate quotes to this article. 


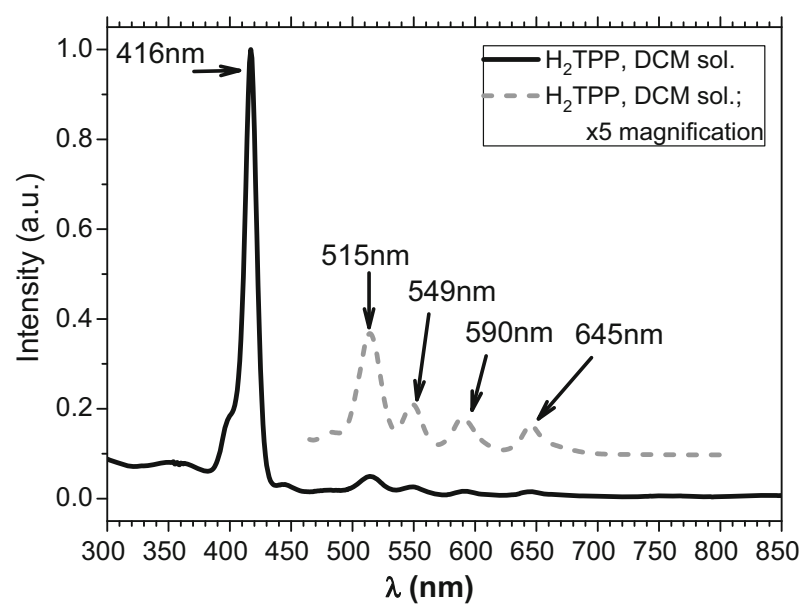

Fig. 6 Normalized absorption spectra of $\mathrm{H}_{2}$ TPP: diluted dichloromethane (DCM) solution (black line); selected $\times 5$ magnification of the Q-bands (DCM solution, dashed gray line) [52]

\section{Results and discussion}

In view of proving that the proposed platform is useful, allowing both RAS and SDR measures, we will show and discuss new experimental results besides the traditional calibration samples for both the systems. In Fig. 7, we show the calibration of the RAS apparatus. Here, we compare signals acquired on $\mathrm{Si}(110)$ and on $\mathrm{Si}(001)$. We also highlight the Soret band region, which is characteristic of the $\mathrm{H}_{2}$ TPP optical properties. Results are in good agreement with data reported in the literature. A signal-to-noise ratio of $10^{-4}$ is easily obtained (a signal of $5 \times 10^{-4}$ is thus detectable), in agreement with Ref. [43], but the performances can be even improved of about an order of magnitude as already reported in our previous studies [31]. By using a $150 \mathrm{~W}$ Xe lamp, by properly change the acquisition time and the software sampling, together with a precise control of the photomultiplier applied voltage, signals as low as $10^{-5}$ become measurable.

Here, conversely, we decided to exploit a stabilized deuterium/halogen lamp in view of adopting the same light source with respect to the two optical configurations. Despite having a different light emission of some order of magnitudes with respect to the traditional Xe lamp, our electronic and software system is able to reach a stability comparable to what already reported in the RAS literature.

SDR calibration is reported in Fig. 8a, where data from HOPG samples either clean (pristine) or covered by a 5-nm-thick $\mathrm{H}_{2} \mathrm{TPP}$ film are compared. The signal from the porphyrin film (full line) is characterized by a clear feature centered at around $430 \mathrm{~nm}$, superimposed to a smooth linear background originating by a not perfect equivalence between the two optical channels (sample and reference), as evidenced by the signal from pristine HOPG (dotted line). The SDR signal-to-noise ratio can be enhanced if a beam splitter [53] is used to obtain two optical paths instead of exploiting brunched optical fibers. Nonetheless, the latter can be easily arranged inside an optical set-up or coupled with molecular beam epitaxy chambers. When compared to the optical spectrum of $\mathrm{H}_{2}$ TPP in dilute solution, reported in Fig. 6, the present data from $\mathrm{H}_{2}$ TPP thin films show a sizable redshift (around $0.15 \mathrm{eV}$ ) accompanied by significant broadening of the Soret band. This result has been attributed to molecule-molecule interaction in the condensed phase [54]. 
Fig. 7 RAS spectra from $\mathrm{Si}(110)$ (full line) and $\mathrm{Si}(001)$ (dotted line) surfaces

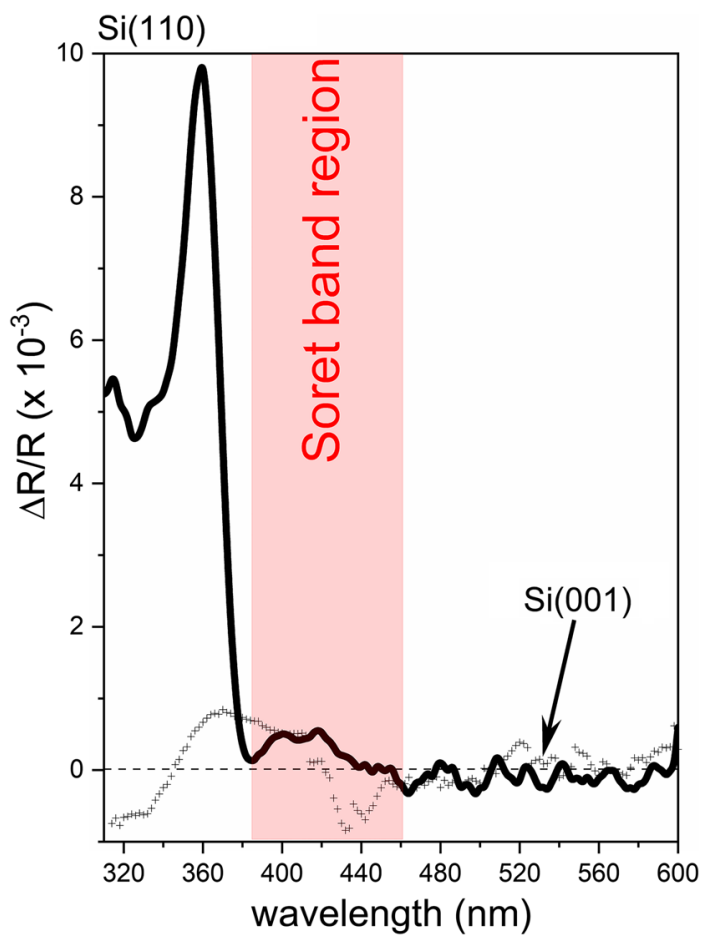

Moreover, polarization-resolved SDR spectra can be acquired by placing a Glan polarizer along the sample-channel of the SDR apparatus. Our results are presented in Fig. 8b, where data corresponding to two orthogonal polarization axes, $\alpha$ and $\beta$, are reported as full and dashdot lines, respectively. In particular, we have selected the configuration which maximizes the RAS anisotropy signal, i.e., with $\alpha$ aligned along the HOPG cleavage direction [55].

It is clearly seen that the SDR spectra of $\alpha$ and $\beta$ polarization are almost superimposable, indicating that the porphyrin film is largely isotropic. This is probably due to an equivalent distribution of $\mathrm{H}_{2}$ TPP domains arranged along different directions. A small difference between the $\alpha$ and $\beta$ spectra is observed in the $420-460 \mathrm{~nm}$ wavelength range, i.e., within the Soret band region. A subtraction of spectrum $\beta$ from $\alpha$ (not reported in the image), which simulates a RAS spectrum that has a different signal definition as reported in Eq. (2), shows a peak placed at around $440 \mathrm{~nm}$. Such a measurement can however be more effectively done by the RAS technique. Figure 8c directly compares the SDR and the RAS spectra acquired on the same sample. The RAS peak (at $440 \mathrm{~nm}$ ) is red-shifted with respect to the main Soret transition as detected by unpolarized SDR $(430 \mathrm{~nm})$.

Our test sample proves that a comprehensive investigation of the optical properties of the thin molecular films (here porphyrins) thus requires the application of both the optical apparatuses. In fact, RAS investigation of complex systems (e.g., molecular clusters or crystals, buried interfaces, etc.) highlights features arising from anisotropies, in turns originating from (possibly small) differences between the reflected signals. SDR, on the other hand, allows to directly measure both the RAS components. 

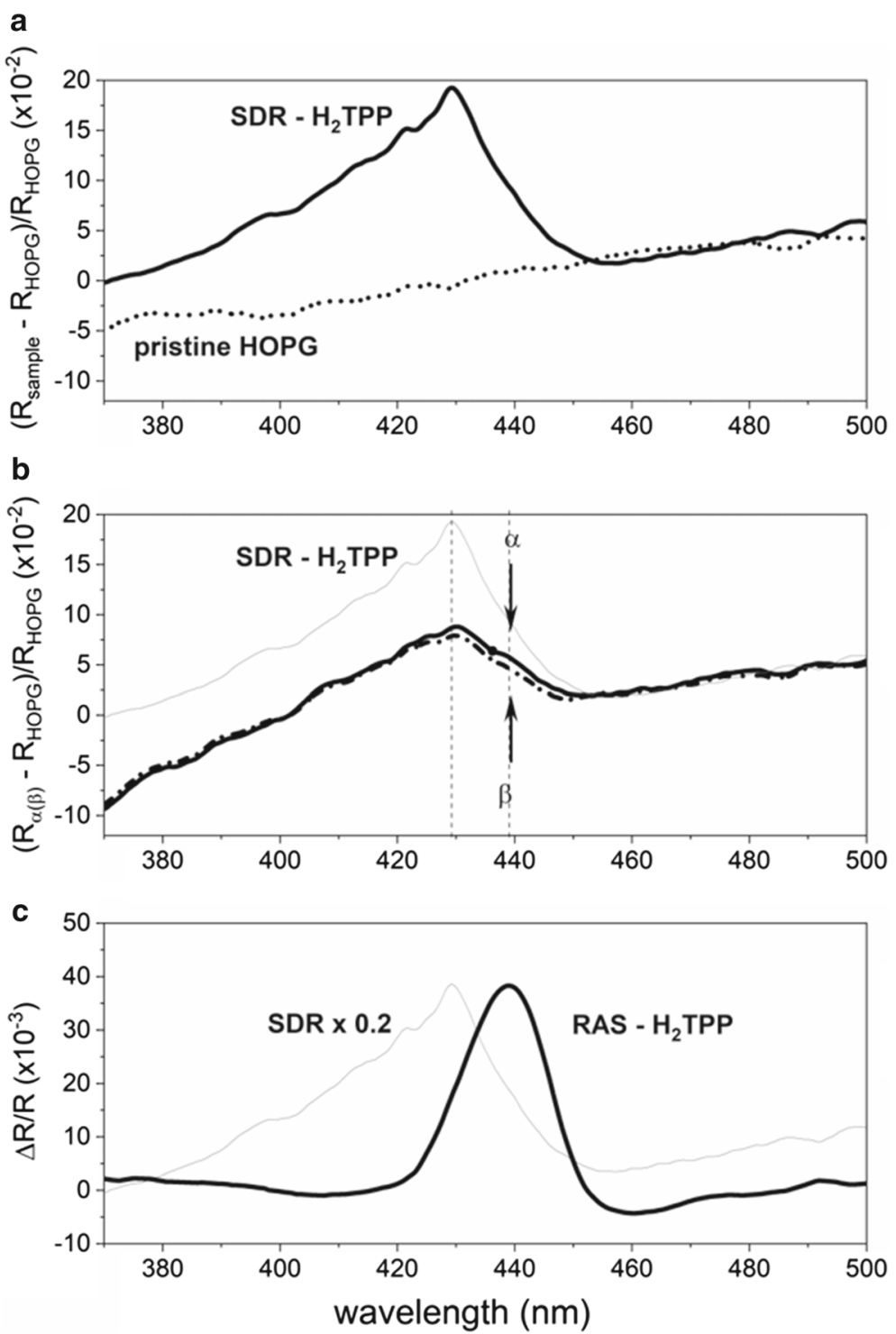

Fig. 8 (a) SDR spectra comparison between a 5 nm-thick $\mathrm{H}_{2}$ TPP film and a pristine HOPG; (b): polarized SDR; (c): comparison between SDR and RAS spectra acquired on the porphyrin thin film

\section{Conclusions}

Optical spectroscopies are, nowadays, widely employed to study surfaces, interfaces, chemical reactions, technological processes, etc. SDR, first used at the end of seventies, is probably the forefather of other surface sensitive optical spectroscopies. RAS was instead described only in the middle of eighties. Nonetheless, the latter has been widely exploited in many researches due to its intrinsic stability and reliability. RAS, by using polarized light, directly reveals the reflectance difference from two orthogonal directions of the studied sample. When 
applied on reconstructed surfaces or ultra-thin films, RAS spectrum is superimposable to that one obtained by SDR in polarized light, as in $\mathrm{Si}(111)-2 \times 1$. In the latter case, polarized SDR reveals a full surface anisotropy (i.e., a null signal when light is polarized perpendicular to the Pandey's chains) in perfect agreement with the anisotropy revealed by RAS. However, when complex, stratified and/or buried interfaces are investigated by RAS, spectra show anomalies such as the "derivative-like" shape observed in porphyrin Langmuir-Blodgett films. The reason is related to the fact that the reflected beam carries the contribution of each layer met along the optical path.

In this work, we propose a combination of both SDR and RAS techniques when such kind of complex organic films must be studied. SDR, carried out under polarized light, can help in understanding where sample anisotropies arise, giving the possibility of a proper interpretation of RAS spectra. The latter, conversely, allows for a fast and more precise evaluation of the relative sample anisotropy, and it is mandatory when very small signals must be detected. SDR and RAS can have some optical parts in common (light source, polarizers, lenses), but the electronic and acquisition schemes are generally different. Despite RAS requires specific devices (namely, the lock-in amplifier), we have presented a viable possibility to build up an affordable electronic architecture and platform that can drive both the SDR and RAS apparatus. Our approach has been applied to a prototypical system: thin $\mathrm{H}_{2}$ TPP grown on HOPG. The authors already showed the RAS signal evolution as a function of the porphyrin film thickness and discussed the difficulty in its interpretation [55]. Here, we prove how the rationale of the optical spectra can be deduced by the combination of SDR and RAS data.

Funding Open access funding provided by Politecnico di Milano within the CRUI-CARE Agreement.

Open Access This article is licensed under a Creative Commons Attribution 4.0 International License, which permits use, sharing, adaptation, distribution and reproduction in any medium or format, as long as you give appropriate credit to the original author(s) and the source, provide a link to the Creative Commons licence, and indicate if changes were made. The images or other third party material in this article are included in the article's Creative Commons licence, unless indicated otherwise in a credit line to the material. If material is not included in the article's Creative Commons licence and your intended use is not permitted by statutory regulation or exceeds the permitted use, you will need to obtain permission directly from the copyright holder. To view a copy of this licence, visit http://creativecommons.org/licenses/by/4.0/.

\section{References}

1. Z. Cao, F. Hu, C. Zhang, S.N. Zhu, M. Xia, X. Wang, Adv. Photon. 2, 054001 (2020)

2. S. Wieghold, L. Nienhaus, Joule 4, 524 (2020)

3. S. Fratini, M. Nikolka, A. Salleo, G. Schweicher, H. Sirringhaus, Nat. Mater. 19, 491 (2020)

4. A. Leonhardt, C.J.L. de la Rosa, T. Nuytten, L. Banszerus, S. Sergeant, V.K. Mootheri, T. Taniguchi, K. Watanabe, C. Stampfer, C. Huyghebaert, S. De Gendt, Adv. Mater. 7, 2000413 (2020)

5. H.-L. Du, W.-H. Zhang, B.-F. Ju, Z. Sun, A. Sun, Rev. Sci. Instrum. 91, 036103 (2020)

6. K. Maier, A. Helwig, G. Müller, M. Eickhoff, Semicond. Gas Sens. 2020, 239 (2020)

7. X. Guo, S. Li, S. Mu, Y. Zhang, X. Liu, Haixia Zhang. Spectrochim. Acta Part A Mol. Biomol. Spectrosc. 226, 117625 (2020)

8. N. Dey, S. Bhattacharjee, S. Bhattacharya, ChemistrySelect 5, 452 (2020)

9. S. Mu, H. Gao, C. Li, Y. Wang, Y. Zhang, C. Ma, H. Zhang, X. Liu, Talanta 221, 121606 (2020)

10. K.Y. Kandurova, E.V. Potapova, E.A. Zherebtsov, V.V. Dremin, E.S. Seryogina, A.Y. Vinokurov, A.V. Mamoshin, A.V. Borsukov, Y.V. Ivanov, A.V. Dunaev, Opt. Spectrosc. 128, 742 (2020)

11. Z. Tang, J. Huang, H. He, C. Ma, K. Wang, Coord. Chem. Rev. 415, 213317 (2020)

12. A.A. Shatskaya, D.N. Artemyev, I.A. Bratchenko, J. Appl. Spec. 87, 112 (2020)

13. J. Jo, E. Jung, J.C. Park, J. Hwang, Curr. Appl. Phys. 20, 237 (2020) 
14. I. Ohlídal, J. Vohánka, M. Čermák, D. Franta, Ellipsometry of layered systems, in Optical Characterization of Thin Solid Films. ed. by O. Stenzel, M. Ohlídal (Springer, Cham, 2018)

15. Z. Li, R. Sun, M.A. Rahman, J. Feng, A. Olah, E. Baer, Polymer 167, 182 (2019)

16. S.I. Quashou, E.F.M. El-Zaidia, A.A.A. Darwish, T.A. Hanafy, Phys. B 571, 93 (2019)

17. A.F. Al-Hossainy, M.R. Eid, MSh. Zoromba, Mater. Chem. Phys. 232, 180 (2019)

18. M.M. Shehata, H. Kamal, H.M. Hasheme, M.M. El-Nahass, K. Abdelhady, Opt. Laser Technol. 106, 136 (2018)

19. L. Rolinger, M. Rüdt, J. Hubbuch, Anal. Bioanal. Chem. 412, 2047 (2020)

20. E. Backus, J. Schaefer, M. Bonn, Angew. Chem. (accepted)

21. S. Trabattoni, S. Tavazzi, R. Yivlialin, L. Duò, F. Ciccacci, G. Bussetti, Appl. Opt. 59, 8175 (2020)

22. P. Chiaradia, G. Chiarotti, S. Nannarone, P. Sassaroli, Solid St. Commun. 26, 813 (1976)

23. M. Palummo, N. Witkowski, O. Pluchery, R. Del Sole, Y. Borensztein, Phys. Rev. B 79, 035327 (2009)

24. A. Cricenti, B. Nesterenko, P. Perfetti, G. LeLay, C. Sebenne, J. Vac. Sci Technol. 14, 2448 (1996)

25. S. Selci, A. Cricenti, F. Ciccacci, A.C. Felici, C. Goletti, Z. Yong, G. Chiarotti, Surf. Sci. 189, 1023 (1987)

26. Y. Borensztein, O. Pluchery, N. Witkowski, Phys. Rev. Lett. 95, 117402 (2005)

27. R. Forker, T. Fritz, Phys. Chem. Chem. Phys. 11, 2142 (2009)

28. A. Bossi, J. Waluk, R. Yivlialin, M. Penconi, M. Campione, G. Bussetti, Chem. Photo. Chem. 4, 5264 (2020)

29. P. Chiaradia, A. Cricenti, S. Selci, G. Chiarotti, Phys. Rev. Lett. 52, 1145 (1984)

30. C. Goletti, G. Bussetti, F. Arciprete, P. Chiaradia, G. Chiarotti, Phys. Rev. B 66, 153307 (2002)

31. G. Bussetti, M. Campione, M. Riva, A. Picone, L. Raimondo, L. Ferraro, C. Hogan, M. Palummo, A. Brambilla, M. Finazzi, L. Duò, A. Sassella, F. Ciccacci, Adv. Funct. Mater. 24, 958 (2014)

32. D.E. Aspnes, A.A. Studna, Phys. Rev. Lett. 54, 1956 (1985)

33. C. Goletti, G. Bussetti, P. Chiaradia, A. Sassella, A. Borghesi, Org. Electr. 5, 73 (2004)

34. A. Sassella, L. Raimondo, L. Fazi, S. Trabattoni, B. Bonanni, G. Bussetti, C. Goletti, Org. Electr. 62, 102 (2018)

35. E.E. Barritt, C.I. Smith, D.S. Martin, K. Gentz, K. Wandelt, P. Weightman, EPL 92, 57005 (2010)

36. C. Goletti, G. Bussetti, A. Violante, B. Bonanni, M. Di Giovannantonio, G. Serrano, S. Breuer, K. Gentz, K. Wandelt, J. Phys. Chem. C 119, 1782 (2015)

37. M. Penconi, L. Ferraro, J. Waluk, L. Duò, F. Ciccacci, A. Bossi, M. Campione, G. Bussetti, Proceedings 56, $44(2020)$

38. J.D.E. McIntyre, D.E. Aspnes, Surf. Sci. 24, 417 (1971)

39. C. Goletti, R. Paolesse, E. Dalcanale, T. Berzina, C. Di Natale, G. Bussetti, P. Chiaradia, A. Froiio, L. Cristofolini, M. Costa, A. D'amico, Langmuir 18, 6881 (2002)

40. C. Castillo, R.A. Vázquez-Nava, B.S. Mendoza, Phys. Stat. Sol. C 8, 2971 (2003)

41. S. Selci, F. Ciccacci, G. Chiarotti, P. Chiaradia, A. Cricenti, J. Vac. Sci. Technol A 5, 327 (1987)

42. A. Salvati, P. Chiaradia, Appl. Opt. 39, 5820 (2000)

43. P. Weightman, D.S. Martin, R.J. Cole, T. Farrell, Rep. Prog. Phys. 68, 1251 (2005)

44. G. Bussetti, M. Campione, A. Sassella, L. Duò, Phys. Stat. Sol. B 252, 100 (2015)

45. O. Plucery, N. Witkowski, Y. Borensztein, Phys. Stat. Sol. B 242, 2696 (2005)

46. R.R. Moore, C.E. Banks, R.G. Compton, Anal. Chem. 76, 2677 (2004)

47. R. Yivlialin, L. Magagnin, L. Duò, G. Bussetti, Electrochim. Acta 276, 352 (2018)

48. R. Yivlialin, M. Penconi, G. Bussetti, A. Orbelli Biroli, M. Finazzi, L. Duò, A. Bossi, App. Surf. Sci. 442, 501 (2018)

49. R.V. Lapshin, Rev. Sci. Instrum. 69, 3268 (1998)

50. B. Wehrle, H.-H. Limbach, M. Köcher, O. Ermer, E. Vogel, Angew. Chem. 26, 934 (1987)

51. M. Schlabach, H.-H. Limbach, E. Bunnenberg et al., J. Am. Chem. Soc. 115, 4554 (1993)

52. C. Goletti, R. Paolesse, C. Di Natale, G. Bussetti, P. Chiaradia, A. Froiio, L. Valli, A. D’Amico, Surf. Sci. 501, 31 (2002)

53. H. Zaglmayr, C.G. Hu, L.D. Sun, P. Zeppenfeld, Meas. Sci. Technol. 25, 115603 (2014)

54. N.J. Hestand, F.C. Spano, Acc. Chem. Res. 50, 341 (2017)

55. G. Bussetti, M. Campione, L. Ferraro, L. Raimondo, B. Bonanni, C. Goletti, M. Palummo, C. Hogan, L. Duò, M. Finazzi, A. Sassella, J. Phys. Chem. C 118, 15649 (2014) 